\title{
Simultaneous Laparoscopic-Assisted Colorectal Resection and Nephrectomy
}

\author{
Seunghun Lee, M.D., Seung-Hyun Lee, M.D., Taeksang Kim, M.D., Sunguhn Baek, M.D., Byungkwon Ahn, M.D.,
} Jooweon Chung, M.D., Eunji Kim, M.D.

Department of Surgery, Kosin University Gospel Hospital, Kosin University College of Medicine, Busan, Korea

Simultaneous laparoscopic surgery for colorectal cancer and coexisting abdominal disease is shown to be feasible. However, simultaneous laparoscopic colorectal resection and nephrectomy is rarely documented, and its feasibility is unknown. We report two cases of simultaneous colorectal resection for colorectal cancer and nephrectomy. In the first case, a 71-year-old female underwent laparoscopic right hemicolectomy for an ascending colon cancer and left nephrectomy for a left non-functioning kidney. The second patient was a 77-year-old male with descending colon cancer and left renal cell carcinoma who underwent laparoscopic left hemicolectomy and left nephrectomy. The body mass indexes were 21.73 and $26.78 \mathrm{~kg} / \mathrm{m}^{2}$, respectively, and operation time was 275 and 395 minutes. Blood loss was 300 and $250 \mathrm{cc}$, and the postoperative hospital stay was 8 and 10 days. In both cases, there was no postoperative morbidity or mortality. Simultaneous laparoscopic resection for colorectal cancer and nephrectomy is a feasible and safe procedure.

Keywords: Laparoscopy, Surgery, Colorectal tumors, Kidney
Received October 12, 2016

Revised December 7, 2016

Accepted February 8, 2017

Corresponding author

Seung-Hyun Lee

Department of Surgery, Kosin

University College of Medicine, 262,

Gamcheon-ro, Seo-gu, Busan 49267,

Korea

Tel: +82-51-990-6462

Fax: +82-51-246-6093

E-mail: gscrslsh@hanmail.net

\section{INTRODUCTION}

Laparoscopic surgery is considered a standard procedure for many conditions. For colorectal cancer, laparoscopic surgery has become a standard surgical option, with many randomized studies of feasibility, safety, and oncologic outcomes. ${ }^{1}$ Recently, several cases of simultaneous minimal invasive surgery have been reported for multiple disease conditions of different organs. ${ }^{2,3}$ Also, simultaneous laparoscopic surgery for colorectal cancer and coexisting abdominal disease has been demonstrated to be feasible without increasing postoperative morbidity. ${ }^{4}$ However, simultaneous laparoscopic colorectal resection and nephrectomy is rarely documented, and its feasibility is unknown. We report two cases of simultaneous colorectal resection for colorectal cancer and nephrectomy (Table 1).

\section{CASE REPORT}

\section{Case 1}

A 71-year-old female was admitted for an adenocarcinoma of the ascending colon. She had a left non-functioning kidney with hydronephrosis (Fig. 1). Her body mass index was 21.73 $\mathrm{kg} / \mathrm{m}^{2}$. The patient underwent laparoscopic right hemicolectomy and left nephrectomy.

A right hemicolectomy was performed first. The patient 
Table 1. Simultaneous laparoscopic colorectal resection and nephrectomy

\begin{tabular}{lcc}
\hline & Case 1 & Case 2 \\
\hline Sex & Female & Male \\
\hline Age (year) & 71 & 77 \\
BMI (kg/m² & 21.7 & 26.8 \\
\hline ASA score & 2 & 2 \\
Diagnosis & & \\
Colorectum & Adenocarcinoma & Adenocarcinoma \\
Kidney & Hydronephrosis & Renal cell carcinoma \\
Sites of organ & & \\
Colorectum & Ascending colon & Descending colon \\
Kidney & Left & Left \\
Stages of colorectal cancer & T3N2MO & T1NOMO \\
Operative time (min) & 275 & 395 \\
Blood loss (cc) & 300 & 250 \\
Postoperative hospital stay (day) & 8 & 10 \\
Complications & - & - \\
\hline
\end{tabular}

$\mathrm{BMI}=$ Body mass index; $\mathrm{ASA}=$ American society anesthesiologists.

was placed in Trendelenburg position with her left side down. Pneumoperitoneum was performed and maintained with a pressure of $12 \mathrm{mmHg}$. An umbilical camera port $(10 \mathrm{~mm})$ for 0 -degree laparoscope was inserted. Three working ports (10 $\mathrm{mm}$ ) were inserted at the right upper, right lower, and left upper portion on the mid-clavicular line. Another working port $(5 \mathrm{~mm})$ was inserted on the left lower portion (Fig. 2). Laparoscopic right hemicolectomy was performed with a lateralto-medial approach. The cecum and ascending colon were mobilized along the white line of Toldt up to the hepatic flexure and medially to the middle portion of the transverse colon with exposure of the superior mesenteric artery and vein. The ileocolic and right branches of the middle colic vessel were clipped and divided proximally. All mesenteric fat and lymph nodes were dissected toward the specimen. Bowel resection and anastomosis were performed extracorporeally by a $5 \sim 6$ $\mathrm{cm}$ umbilical incision. Anastomosis was performed with the side-to-side technique using linear staplers.

A urologic surgeon performed the next part of the operation. The patient was placed in the right lumbotomy position. An additional port $(10 \mathrm{~mm}$ ) was inserted in the epigastric position (Fig. 2). The descending colon was medially mobilized by dissecting the white line of Toldt, exposing the anterior aspect of the left kidney. The ureter was followed to the hilum of the kidney. $\mathrm{Re}^{-}$ nal vessels were clipped and divided. Dissection was performed

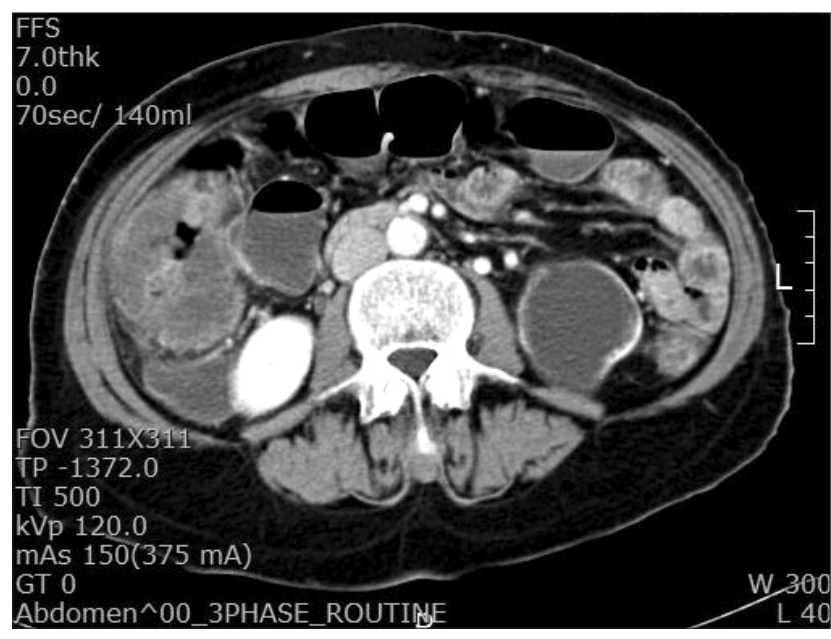

Fig. 1. Computed tomography scan shows a huge tumor with wall thickening at the ascending colon and a large cystic, non-functioning left kidney.

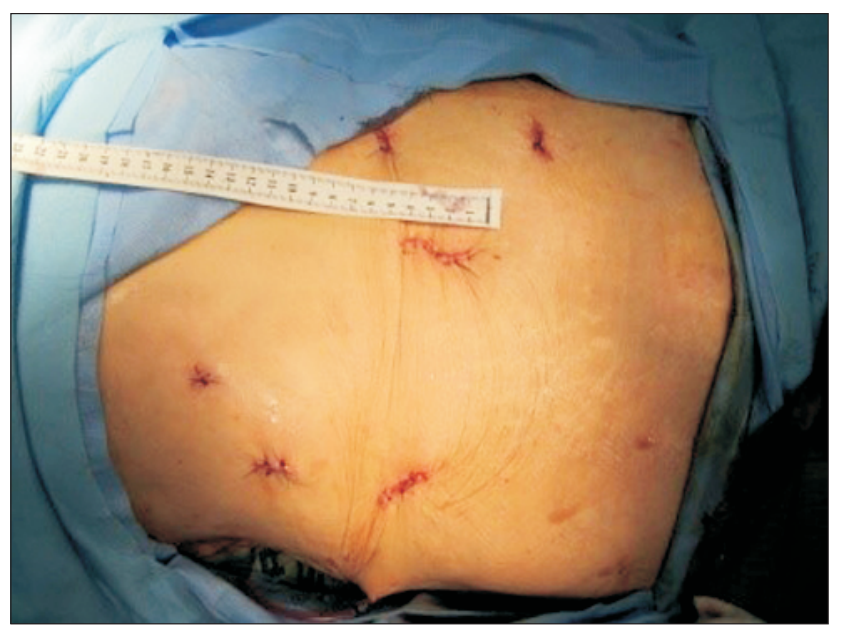

Fig. 2. Operative wound after laparoscopic right hemicolectomy and left nephrectomy.

to mobilize the left kidney with the adrenal gland, perinephric fat tissue, and Gerota's fascia. The entire specimen was placed into an endoscopic bag. Total operation time was 275 minutes, and blood loss was $300 \mathrm{cc}$. The patient was discharged on the eighth postoperative day without postoperative complication.

\section{Case 2}

A 77-year-old male with early descending colon cancer and left renal cell carcinoma (Fig. 3) underwent laparoscopic left hemicolectomy and left nephrectomy. His body mass index was $26.78 \mathrm{~kg} / \mathrm{m}^{2}$.

The patient was placed in Trendelenburg position with his right side down. The colorectal surgeon performed the lapa- 


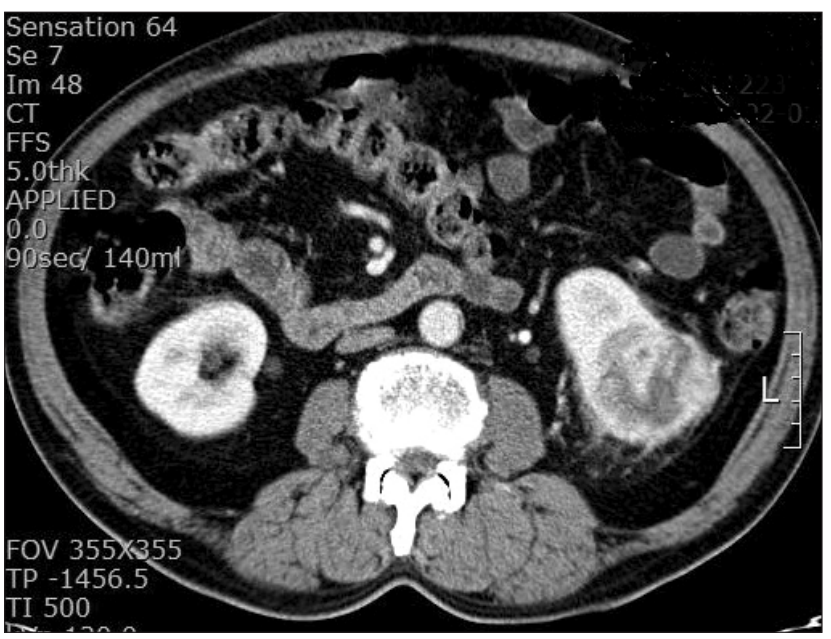

Fig. 3. Computed tomography scan shows left renal cell carcinoma.

roscopic left hemicolectomy first, and then the urologic surgeon performed the left nephrectomy with the same technique as in Case 1. The trocar port sites were the same as in Case 1. Medial-to-lateral retroperitoneal dissection of the mesocolon and early division of the left colic and left branches of the middle colic vessel were performed with preservation of the inferior mesenteric artery and vein and lymph node harvest. Extracorporeal colon resection and side-to-side anastomosis using two linear staplers were performed. The operation time was 395 minutes. Central obesity, adhesions, and a short mesentery requiring extended mobilization of the left colon may have accounted for the longer operation time than required for Case 1. Blood loss was $250 \mathrm{cc}$. The postoperative hospital stay was 10 days. There was no postoperative morbidity or mortality.

\section{DISCUSSION}

A few cases of simultaneous laparoscopic surgery have been reported for synchronous colorectal and renal disease on the same side of the abdomen. ${ }^{5-7}$ Veenstra et al. ${ }^{6}$ and O'Sullivan et $\mathrm{al}^{7}$. reported feasible simultaneous laparoscopic surgery on the contralateral side of the abdomen. The present report describes two cases of ipsilateral and contralateral abdominal procedures.

Popular use of imaging studies, such as computed tomography, magnetic resonance imaging, and positron emission tomography, may be related to a high incidence of multiple primary malignancies. Aydiner et al. ${ }^{8}$ reported that $1 \%$ of patients with carcinoma had multiple primary malignancies. In the Asian population, the stomach is the most common site of multiple primary malignancies with colorectal cancer. ${ }^{9}$ The frequency of multiple primary colorectal and gastric cancers is reported to range between $0.8 \%$ and $3.9 \%$. Synchronous re- nal cell carcinoma with colorectal cancer is rare, but may be found in up to $4.85 \%$ of colorectal cancer cases. ${ }^{10}$ Therefore, surgeons must decide between simultaneous surgery or staged surgery for synchronous multiple primary malignancy.

Simultaneous surgery for synchronous disease of different organs has several disadvantages, including longer operative time, more blood loss, higher cardiopulmonary distress risk, and challenging operative technique. However, this technique provides patients with various benefits, such as shortened hospital stay, less postoperative pain, and better cosmesis. More research is needed to confirm the oncologic outcome of synchronous tumor resection. Simultaneous laparoscopic colorectal surgery and nephrectomy is a feasible and safe procedure.

\section{REFERENCES}

1) Jayne DG, Thorpe HC, Copeland J, Quirke P, Brown JM, Guillou PJ. Five-year follow-up of the Medical Research Council CLASICC trial of laparoscopically assisted versus open surgery for colorectal cancer. Br J Surg 2010;97:1638-1645.

2) Suh HH, Cho YK, Rheu HG. Synchronous Cholecystectomy and Totally Extraperitoneal (TEP) Herniorrhaphy Using an Umbilical Incision. J Minim Invasive Surg 2012;15:79-82.

3) Choi SH, Lee SH, Ahn BK, Baek SU. Single-incision Laparoscopic Surgery for Simultaneous Appendectomy and Cholecystectomy. J Minim Invasive Surg 2014;17:51-54.

4) Zhu QL, Zheng MH, Feng B, et al. Simultaneous laparoscopyassisted low anterior resection and distal gastrectomy for synchronous carcinoma of rectum and stomach. World J Gastroenterol 2008;14:3435-3437.

5) Kim SH, Park JY, Joh YG, Hoe HE. Simultaneous laparoscopic radical nephrectomy and laparoscopic sigmoidectomy for synchronous renal cell carcinoma and colonic adenocarcinoma. J Laparoendosc Adv Surg Tech A 2004;14:179-181.

6) Veenstra GM, Fossion LM, de Laet K, Luijten AA. Synchronous Laparoscopic Radical Nephrectomy Left and Contralateral Right Hemicolectomy during the Same Endoscopic Procedure. ISRN Surg 2011;2011:179456.

7) O’Sullivan M, Kearney DE, Giri SK, Coffey JC. Combined laparoscopic-assisted nephrectomy and complete mesocolic excision for synchronous renal and colon cancers. BMJ Case Rep 2015;2015.

8) Aydiner A, Karadeniz A, Uygun K, et al. Multiple primary neoplasms at a single institution: differences between synchronous and metachronous neoplasms. Am J Clin Oncol 2000;23:364-370.

9) Lee JH, Bae JS, Ryu KW, et al. Gastric cancer patients at highrisk of having synchronous cancer. World J Gastroenterol 2006;12:2588-2592.

10) Halak M, Hazzan D, Kovacs Z, Shiloni E. Synchronous colorectal and renal carcinomas: a noteworthy clinical entity. Report of five cases. Dis Colon Rectum 2000;43:1314-1315. 